\title{
Efficacy of injections of phosphatidylcholine into fat deposits-a non-surgical alternative to liposuction in body- contouring
}

\author{
Heinrich Karl-G \\ Kosmetische Chirurgie DDr.Heinrich, Vienna, Austria
}

Address for correspondence: Kosmetische Chirurgie, Heinrich Karl-G, Vienna, Austria. E-mail: kunden@ddr-heinrich.at

\section{ABSTRACT}

Injecting phosphatidylcholine has been used in South America as a non-surgical treatment in body contouring. The objective of this study was to demonstrate the efficacy of injecting phosphatidylcholine in the reduction of localised fat deposits. 86 patients were included in the study. Patients received 13 treatments in localised fat deposits in various areas of the body using phosphatidylcholine. After treatment with phosphatidylcholine $(250 \mathrm{mg} / 5 \mathrm{ml})$, fat deposits show an average circumferential reduction per application of $2.70 \mathrm{~cm}$. No patient showed irregularities, dimples or any serious side effect after treatment. Results remained stable during the time of follow up. All patients showed remarkable reductions of the fat deposits treated with phosphatidylcholine. Using the correct technique, injecting phosphatidylcholine may be a safe and efficacious alternative to liposuction in patients objecting to surgery.

\section{KEY WORDS}

Injection lipolysis, Fat-away-injection, Lipostabil, Phosphatidylcholine, Liposuction, Microcannula, Cosmetic surgery

\section{INTRODUCTION}

lthough tumescent liposuction with microcanulas ${ }^{1}$ has been proven as a highly efficient method in correcting unwanted localised fat deposits with few side effects, some patients object to surgery. Anecdotal evidence from both South-America and Europe states that injecting phosphatidylcholine might be a safe and efficacious way to correct localised fat deposits.

Phosphatidylcholine (a phospholipid) is a bile component and is responsible for lipid emulsification from the diet. ${ }^{2}$ Phosphatidylcholine is widely used in prophylaxis and treatment of fat embolism. It has also been shown to reduce the systemic levels of cholesterol and triglycerides. ${ }^{3,7}$ Bobkova et $a l^{4}$ showed that upon increasing phosphatidylcholine, cell membranes improved their receptor properties, augmented their sensitivity to insulin, and accelerated lipolysis. Phosphatidylcholine has also been employed intravenously in patients with cardiac ischemia. ${ }^{5}$ A marked reduction of atheromatous plaques with a reduction in plasma cholesterol occurs. Rittes ${ }^{6}$ described the local injection of phosphatidylcholine to reduce the size of infraorbital fat pads, thus offering an alternative to surgical correction. Long-term follow-up of patients treated with phosphytidlycholine injections to reduce local fat 
deposits indicate that the reduction of fat deposits is permanent, provided weight gain is low or none. ${ }^{8}$

The objective of this study was to evaluate and demonstrate the efficacy of injecting phosphatidylcholine as a nonsurgical treatment for the correction of localised fat deposits.

\section{PATIENTS AND METHODS}

86 patients ( 82 females, 4 males) between 21 and 68 years were included in the study (Mean: 41.05; Std. Dev.: 11.16). Informed consent was obtained from all individuals. Pretreatment evaluation ensured that every patient was in good health (ASA-Class 1-2). Patients with lymphedema or periodical swellings due to lymphatic problems were excluded from the study.

For the study, patients received treatment on 1 specific

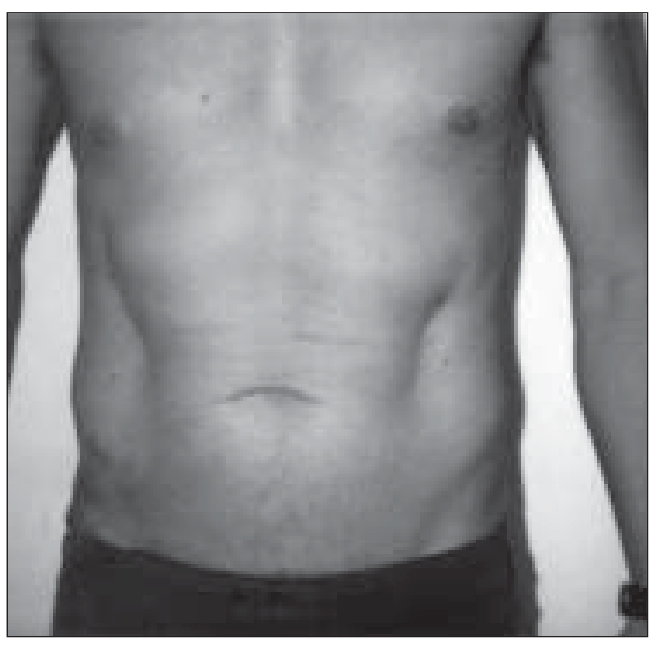

Figure 1A: Patient before injection lipolysis

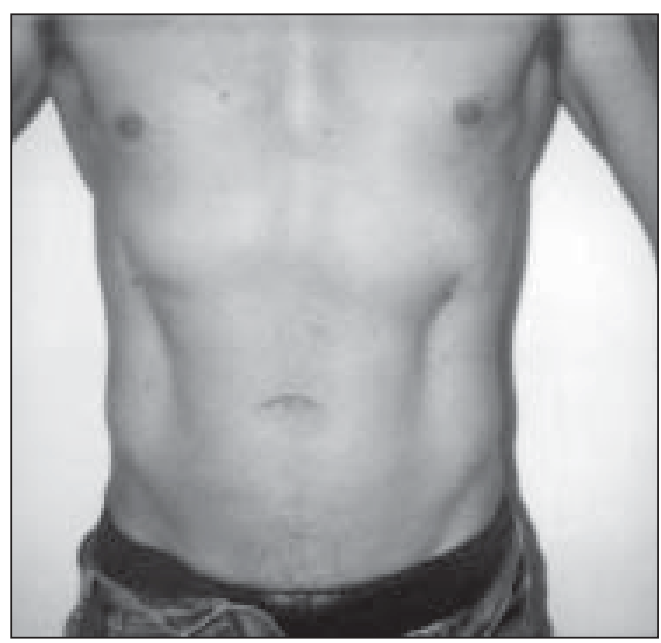

Figure 1B: Same Patient after treatment area, each treatment included 1 to 3 applications. One specific area included for example, left and right hip or left and right side of the belly, etc. The areas treated were chest, hips, waist, thighs (outer, inner, front), knees, arms, calf, flanks, belly (upper, lower), buttocks, mons veneris and back. The size and location of the fat deposits was examined and the circumference was measured. It was ensured that the measurement of fat deposits before and after application took place on exactly the same points. In addition, photographs were taken before and after the procedure to document the changes resulting from treatment.

The injections were performed in an outpatient setting with the patient lying on the operating table, injecting phosphatidylcholine in an ambulatory setting. After the marking of the fat deposits, the skin was anaesthetised using a topical lidocain preparation. Using 30-gauge needles of $1.2 \mathrm{~cm}$ length, phosphatidylcholine $(250 \mathrm{mg} / 5 \mathrm{ml})$

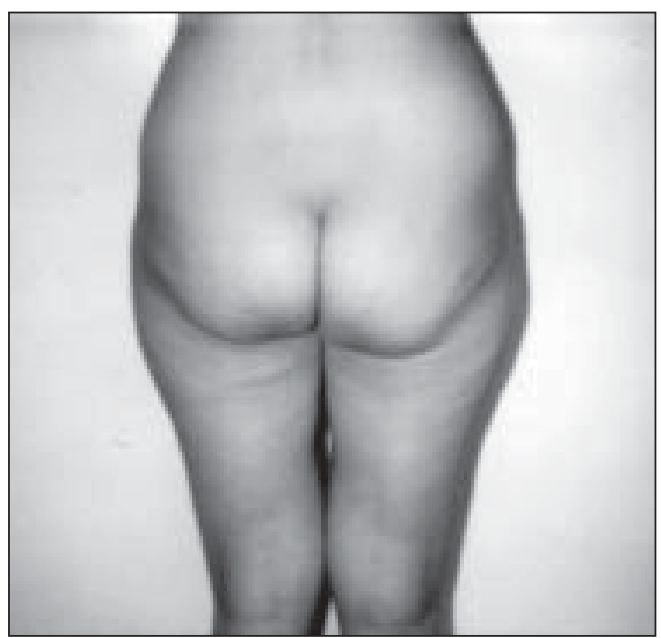

Figure 2A: Patient from the Viennese injection lipolysis study before treatment

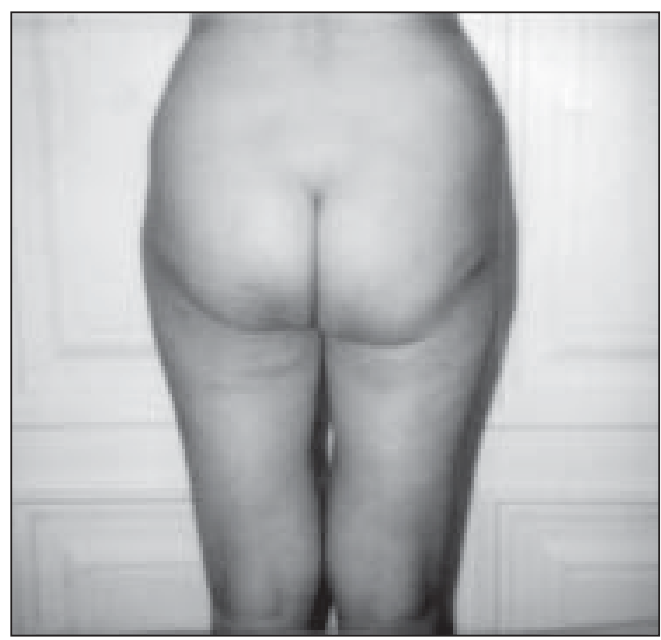

Figure 2B: Patient after injection lipolysis 
was injected into the fat deposit following a multiinjection pattern according to the shape of the fat deposit, Syringes and needles used for injecting phosphatidylcholine). The amounts of phosphatidylcholine injected into each fat deposit ranged between $5 \mathrm{ml}$ and $30 \mathrm{ml}$ per application according to the individual needs of the patient.

The result of every single application per patient and area was monitored by measuring the most prominent circumference of the treated area before the application. 14 days after the application the same points on the fat deposits were used for circumferential measurement. To enhance the cosmetic result, some patients received an additional application 14 days after the first application. A few patients also received a third application 14 days after the second.

Patients were asked to hold their body-weight stable during the time of treatment to ensure that reduction of fat deposits was not due to weight loss. No other type of treatment of fat deposits (e.g. liposuction, mesotherapy, vigorous massaging) was allowed during the study period. Patients who lost or gained weight during the time of the study or did not comply with the conditions mentioned above were excluded from the sample.

\section{RESULTS}

\section{Reduction of fat deposits}

Circumferential reductions of the treated fat deposits between $0.0 \mathrm{~cm}$ and $14.5 \mathrm{~cm}$ per application were achieved. The average circumferential reduction per application was $2.41 \mathrm{~cm}$ [Figure 1], Patient before and 2 weeks after second treatment and [Figure 2], Patient before and 2 weeks after second treatment).

\section{Statistical evaluation}

For the statistical evaluation of the reduction, the difference in measurements for each specific area before and after treatment was calculated (measurement after treatment minus measurement before treatment). This resulted in one to eleven values per patient, depending on the number of areas treated and the number of applications; a total of 257 values occurred for the sample of $\mathrm{N}=86$. A mean reduction of $-2.41 \mathrm{~cm}$ was achieved (standard deviation: 1.62; lower confidence level (95\%): -2.61; upper confidence level (95\%): -2.21). The one-sample students t-test is highly significant $(t=-23.834 ; p<0,0001 ; d f=256)$. Therefore, the mean circumferential reduction of $2.41 \mathrm{~cm}$ can be seen as significant.

[Table 1], Mean circumferential reduction of specific areas, shows the mean circumferential reductions for the specific areas treated.

In 13 cases no reduction was measured $(0 \mathrm{~cm})$, corresponding to a non-responder-rate of $5.06 \%$. The rate of low-responders with reductions of less than $1 \mathrm{~cm}$ per treatment is $6.23 \%$ (16 cases), whereas the rate of highresponders (reductions of more than $3 \mathrm{~cm}$ per treatment) equals $18.29 \%$ ( 47 cases).

Table 1: Mean circumferential reduction of specific areas

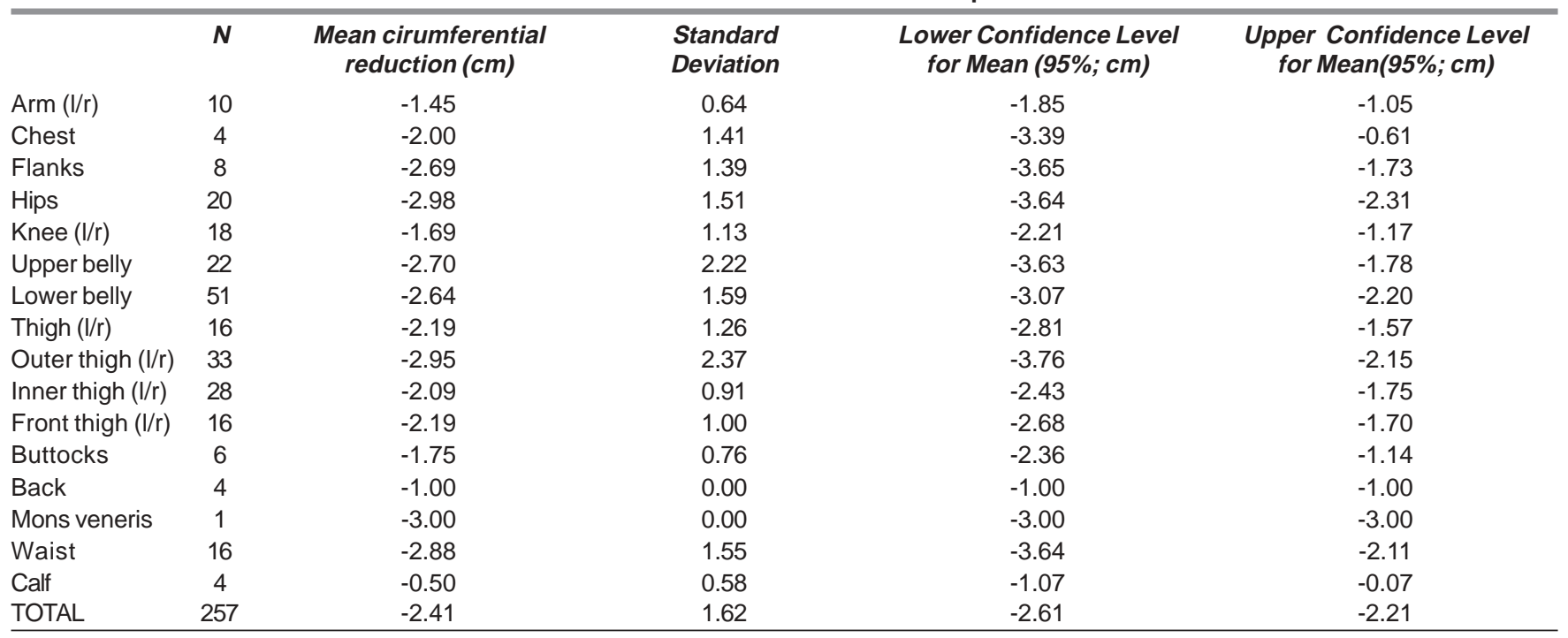




\section{Side effects}

Cosmetic improvement was seen in every patient: no irregularities or dimples were seen; no serious side effects or complications were noted. All patients reported some pain, swelling and erythema, which typically lasted for 2-3 days. Haematomas also occurred in some patients. The study was performed from October 2003 until May 2004. During this time, no re enlargement of fat deposits was reported.

\section{DISCUSSION}

Injecting phosphatidylcholine (made from soy bean-oil) seems to offer a non-surgical alternative to liposuction in patients objecting to surgery. It appears to be safe and effective in the correction of fat deposits. To ensure a smooth reduction of the fat deposit it is important to achieve the adequate and necessary concentration of phosphatidylcholine in every part of the treated area, thus requiring a specific multi-injection technique.

\section{Need for further studies}

While significant reduction of fat deposits was seen in all patients participating in the study, some patients did not seem to respond as well as the others. The reason for this different response could be a lower sensibility of the fat cells of these patients to phosphatidylcholine. Further studies need to be done to evaluate this hypothesis. Evidence from South America indicates that reductions of fat deposits are permanent and that no long-term side effects have been seen in thousands of patients treated in the past years - this also needs to be verified in further studies.

\section{CONCLUSION}

In the opinion of the author, the efficacy of phosphatidylcholine injections in the reduction of localised fat deposits is proven. To ensure a smooth and satisfying cosmetic result it is critical to use the correct injection technique. This method is no substitute of tumescent liposuction as "state of the art" in body contouring. However, in the hands of the skilled aesthetic surgeon, it could offer an alternative to liposuction for patients with small and medium sized fat deposits who do not want to undergo surgery. More studies have to be done to assess the anecdotal evidence from South America that reduction after treatment is permanent and that no long-term side effects have been seen in thousands of patients.

\section{REFERENCES}

1. Klein J. Tumescent Technique \& Microcannular Liposuction. Mosby 2000.

2. Montgomery R, Conway T. Bioquímica, $5^{\text {th }}$ edn. 1996. p. 249.

3. Navder KP, Baraona E, Lieber C. Polyenyphosphatidylcholine decreases alcoholic hyperlipemia without affecting the alcoholinduced rise of HDL cholesterol. Life Sci 1997;61:1907.

4. Bobkova VI, Lokchina LI, Korsunsk BH, Tamamova GV. Metabolic effect of lipostabiforte. Kardiologia 1989;29:57.

5. Pogozheva AV, Bobkova SN, Samsonov, MA, Vasilév AV. Comparative evaluation of hypolipidemic effects of omega-3 polyunsaturated acids and lipostabil. Vopr Pitan 1996;4:31.

6. Rittes P. The Use of Phosphatidylcholine in the Correction of Lower Lid Bulging Due to Prominent Fat Pads. Dermatol Surg 2001;27:391-2.

7. Warembourg $\mathrm{H}$, Jaillard J. Experimentation clinique du lipostabil dans le traitement des angiopathies diabetiques. Lille Med XIII 1968:721-3.

8. Rittes P. The Use of Phosphatidlycholine for Correction of Localized Fat Desposits. Aesth Plast Surg 2003;27:315-8. 